\title{
Beneficios del juego en la acción pedagógica
}

\author{
Cristina C. Achavar Valencia ${ }^{1}$
}

Recibido 10 de agosto de 2019 - Aceptado 30 de octubre de 2019

\section{RESUMEN}

A pesar de la importancia del juego para la educación inicial, no ha sido atendido en su justa dimensión en términos investigativos y de desarrollo académico. Si bien existe literatura que trata sobre su importancia metodológica, no se ha relevado en el contexto de la didáctica y la acción pedagógica. Se asume que el juego es una actividad natural de la infancia y que responde a las motivaciones de los niños y niñas para satisfacer la necesidad de entretenimiento; sin embargo, la actividad lúdica reviste ventajas adaptativas sobre otras conductas y constituye una fuente de aprendizaje permanente. El presente artículo expone una reflexión en torno a los beneficios del juego en la acción pedagógica, considerando que el aprendizaje en la infancia temprana configura una etapa esencialmente lúdica. Se concluye que el juego puede adquirir un carácter de espacio cultural, una actividad y una fuente de aprendizaje, trascendiendo a la limitación de entretenimiento o de herramienta o técnica pedagógica.

Palabras clave: juego, infancia, acción pedagógica.

\section{Benefits of Play in Pedagogical Action}

\section{ABSTRACT}

Despite the importance of play in early education, it has not been addressed sufficiently in terms of research and academic development. While there is literature that deals with its methodological importance, it has not been relieved in the context of teaching and pedagogical action. It is assumed that play is a natural childhood activity and responds to children's motivation to meet the need for entertainment, however, recreational activity has adaptive advantages over other behaviors and is a source of lifelong learning. This article presents a reflection on the benefits of play in pedagogical action, considering that early childhood learning sets up an essentially playful stage. It is concluded that play can become a cultural space, an activity and a source of learning, which transcends the limits of entertainment or pedagogical tool or technique.

Key words: play, childhood, pedagogical action.

I Licenciada en Educación y Educadora de Párvulos, Pontificia Universidad Católica de Chile. Diplomada en Enseñanza del Pensamiento PUC. Magíster (C) Estudios Cognitivos, Universidad de Chile. Doctora en Lingǘstica, Pontificia Universidad Católica de Valparaíso. Académica Escuela de Educación Parvularia, Universidad Católica Silva Henríquez. Contacto: cachavarv@ucsh.cl 


\section{Introducción}

El concepto de "juego" guarda relación con la utilización productiva del tiempo y se establece como un objeto propio de nuestro patrimonio cultural, ya que es una capacidad creadora del ser humano que construye realidad (Borghi, 2012). Una de sus funciones principales es, en su mayor parte, "una lucha por algo o una representación de algo. Ambas funciones pueden fundirse, de suerte que el juego represente una lucha por algo o sea una pugna para ver quién reproduce mejor algo" (Huizzinga, 2012, p. 33). Por esta razón es que ha sido estudiado desde diversas perspectivas, atribuyéndole utilidades sicológicas, sociales y educativas. En este último aspecto, la pedagogía ha destacado sus múltiples beneficios en cuanto a la potenciación de la exploración, la autonomía y las relaciones sociales. No obstante, es necesario profundizar en aspectos como el rol del adulto en la actividad lúdica, los tipos de juego que son propios de cada nivel de desarrollo y la importancia del juego como fuente de aprendizaje. En este último punto se centra el presente artículo, que tiene por objetivo exponer la relevancia del juego en el contexto de la primera infancia, a partir de revisión bibliográfica y reflexión pedagógica.

\section{Breve retrospectiva en el estudio del juego}

Los primeros escritos sobre la importancia del juego se remontan a Platón, quien considera que el juego es una forma de entrenamiento de habilidades. Así, señalaba que era necesario "dar alas a los niños desde su primera infancia, a fin de que, cuando sea preciso, se retiren en vuelo". En ese sentido, homologa el juego al aprendizaje, ya que lo considera como el ejercicio de habilidades importantes para un ciudadano.

Posteriormente, precursores de la educación inicial, como Rosseau, Pestalozzi y Fröebel, conceptualizan al juego como una vía de conocimiento. En el caso del primero, propone que la infancia es una etapa clave en la vida humana y enfatiza el rol de la actividad automotivada. Pestalozzi destaca la importancia de la interacción con objetos durante la primera infancia como vía para la adquisición 
de aprendizaje. Fröebel es quien releva el rol del juego como construcción de aprendizaje, ya que postula que éste constituye una expresión espontánea con profundo significado, que media en la construcción del sentido de la realidad. Es mediante el juego cómo los niños y niñas internalizan la cultura y desarrollan su propia identidad.

Otra connotación relativa al juego proviene de las teorías biológicas que explican el comportamiento de los seres vivos. Para este enfoque, el juego es instintivo y tiene el objetivo de contrastar la conducta lúdica con su correlato real. Congruente con lo anterior, Gross (I898, en Smith, 2010), señala que el juego es una práctica previa a las tareas esenciales que denota la vida humana, y adhiere al supuesto de que el juego satisface las necesidades biológicas del ser humano. Desde la psicología, el juego fue considerado por Freud a partir de una perspectiva psicoanalítica. Su teoría propone que el juego es expresión de aquellos aspectos reprimidos por la sociedad, o bien es visto como una compensación de la ansiedad y los traumas, ya que se visualiza como un espacio libre, seguro y sin límites.

Vigostky (I966), por su parte, observó una unidad afectiva en el juego como la realización, mediante la imaginación de deseos irrealizables. No obstante, dicho fenómeno se encuentra ligado al desarrollo cognitivo, ya que el juego de roles (o de simulación) permite al niño ingresar al mundo de las ideas desde referentes concretos. Esto es la transposición desde la experiencia con los objetos hacia el pensamiento. El contexto del juego de simulación exige abstracción en cuanto al cumplimiento de un guión narrativo y escénico que involucra a los participantes.

Piaget (1990), considera que, mediante el juego, los niños representan sus esquemas ya establecidos y se adaptan a la realidad. El juego, por lo tanto, es tributario del desarrollo del pensamiento, puesto que constituye una posibilidad de ejercer sus funciones cognitivas en situaciones reales.

Desde el enfoque antropológico, el juego es cultura, puesto que, más allá de satisfacer una necesidad, entronca al hombre con el conocimiento y la tradición cultural, es decir, es una función humana tan fundamental como la reflexión. En otras palabras, la construcción 
de la cultura surge en forma de juego, ya que "en sus fases primarias, tiene algo de lúdica, es decir, que se desarrolla en las formas y con el ánimo de un juego" (Huizinga, 2012, p. 8I). Si bien el juego connota con un espacio de libertad, de todas formas,

se desarrolla dentro de unos límites temporales y espaciales determinados, según reglas absolutamente obligatorias, aunque libremente aceptadas, acción que tiene su fin en sí misma y va acompañada de un sentimiento de tensión y alegría y de la conciencia de ser de otro modo que en la vida corriente. (Huizinga, 2012, p. 55).

La acción lúdica supone un acuerdo que, si es infringido, constituye la destrucción del mundo mágico. Según Huizinga (2012), los infantes juegan reconociendo el estatus de seriedad y plenitud, es decir, profesan respeto del rol que asumen. El juego crea orden. Está en permanente tensión. Esto último quiere decir que pone en evaluación las habilidades de los jugadores, como la fuerza, la habilidad o la destreza. El orden y la tensión conllevan a la existencia de reglas del juego, lo que se transfiere ulteriormente en la conducta ciudadana y la vida en comunidad.

\section{El juego como actividad de aprendizaje}

El juego es una actividad instintiva e imitativa, y prepara las capacidades para la vida adulta, por lo tanto, es inherentemente un proceso de aprendizaje (Michnick, Hirsh-Pasek \& Singer, 2006), porque provee las claves para comprender los significados de la cultura, así como también la resolución de problemas (Bruner, 1973). Por ende, es una condición de desarrollo, ya que, "en sus diversas manifestaciones, favorece el crecimiento, la afirmación de uno mismo, la confianza en los demás, la curiosidad, el deseo incesante de explorar, la creatividad” (Borghi, 2010, p. I44).

El juego promueve el comportamiento cooperativo y el sentido social, así como también:

is also critical to self-regulation and children's ability to manage their own behavior and emotions. 
es también crítico en términos de importancia para los infantes en cuanto a su autoregulación, así como para el desarrollo de habilidades que permiten manejar su propio comportamiento y sus emociones. (Michnick, Hirsh- Pasek \& Singer, 2006, p. 24)

El juego es un cometido que influye en procesos de autoorganización y desarrollo que contribuyen en la cognición. Supone la coordinación sensorio-motriz, base de la inteligencia y la cognición. Desde esta perspectiva encarnada, el significado se construye en circunstancias activas en el mundo y el entorno, es decir emerge de complejos procesos de acoplamiento estructural. La cognición depende de las experiencias originadas en la posesión de un cuerpo con aptitudes sensorio-motrices, que "están encastradas en un contexto biológico, psicológico y cultural más amplio" (Varela, Thompson \& Rosch, I997, p. 203), es decir; "no es posible hablar del cuerpo como un objeto o en su defecto decir que se tiene un cuerpo, sino que se es un cuerpo" (Toro \& Sabogal, 2017, p. 38). Por tanto, los cimientos de la conducta y naturaleza humana se basan en la corporeidad y la posibilidad de movimiento. En ese contexto, el juego entraña embeberse en el mundo y situarse plenamente en la acción, ya que provee las posibilidades ciertas de participación en el mundo desde lo experiencial, lo que lo convierte, más que en una metodología o técnica pedagógica, en una forma de acoplamiento vital en la realidad. Se extiende como un espacio de construcción centrada en el sujeto, por lo que no se reduce a una mera herramienta educativa, sino que en

un hacer determinado y concreto desde donde el niño vive. La ludicidad no es algo que haya que intencionar, sino algo que hay que permitir y legitimar, pues de esa manera estaremos legitimando, también, la forma en la que los niños se acercan al conocer, tanto de ellos mismos como del entorno en el que viven. (Moreno, 2017, p. 22).

De lo anterior se desprende la relación que se establece entre juego y lenguaje, ya que la experiencia lúdica supone un intercambio con otros que exige el uso de recursos lingüísticos que sostengan la interacción. Dickinson and Tabors (200I) señalan que existen vínculos entre práctica lúdica y adquisición del lenguaje, ya que el vocabulario 
se incrementa conforme se establecen diálogos e intercambios comunicativos. Otro de los aspectos que se ve beneficiado es la alfabetización temprana, ya que muchas de las experiencias que enriquecen dichos conocimientos emergentes acerca de la lectura y escritura, se dan en contexto de juego. Una de las aproximaciones que constituye un predictor importante en la adquisición de la alfabetización es la comprensión del sentido de lo impreso, es decir, el conocimiento de cómo funcionan los textos y cuál es su propósito. En este contexto, la exploración de material impreso de diversa naturaleza provee a los niños y niñas de prácticas escritas útiles para desarrollar la "print awareness" o "conciencia de lo impreso". Se ha demostrado que, al estar en posesión de herramientas y accesorios como libretas de notas, recibos, boletas, recetarios médicos, y utilizarlos con los propósitos establecidos para cada tipo de texto, los estudiantes han incrementado su conocimiento acerca de la tipología de los géneros textuales y la producción de textos en torno a juegos en que se utilizan dichos recursos (Neuman \& Roskos, 1992).

A pesar de su naturaleza espontánea, el juego posee regularidades y consistencia, ya que se propone un acuerdo tácito entre los participantes de la existencia de ciertas reglas que son compartidas, ya que "aquello que se manifiesta no es lo que aparenta ser. Es esta actitud no literal la que permite que el juego presente unas consecuencias amortiguadas: en efecto, permite al juego ser juego" (Garvey, 1985, p. 19). Se establece entonces un pacto o contrato social que permite asumir la existencia de mundos compartidos por la comunidad que participa en la actividad lúdica. El establecimiento de normas y la libertad de actuar conforme a ellas conduce a un estado de satisfacción en los niños y niñas, ya que controlan los límites en los que desarrollan sus acciones. Dichos estados emocionales positivos implican el desarrollo de las áreas atencionales del cerebro y conllevan al proceso de aprendizaje. Esto último guarda relación con la activación del circuito dopaminérgico implicado en la evaluación de la situación ambiental (FernándezEspejo, 2000) y la recepción de recompensa. La actividad lúdica potencia el estado emocional positivo y promueve por tanto el aprendizaje. Esto último implica que la situación de juego es una condición sine qua non en la vida cotidiana de los niños, para 
favorecer un clima de aprendizaje positivo. La motivación deriva de tales estados emocionales.

\section{Conclusiones}

I. El juego puede ser visto como un espacio cultural, una actividad y una fuente de aprendizaje.

2. Desde la perspectiva del juego como espacio, constituye un ámbito vivencial para niños y niñas, en el que encuentran seguridad y libertad para expresar sus emociones, necesidades, estados e intereses. Dicho espacio proporciona los elementos prácticos que conducen a la maduración emocional y social, así como también a la expresión de aspectos que no se conceptualizan en la conciencia (traumas, aprendizajes implícitos, etc.).

3. Desde el punto de vista del juego como actividad, este constituye la esencia del conocimiento del entorno, puesto que pone en práctica mecanismos de imitación y contrastación con la conducta real. Posiciona la acción lúdica como una fuente de discernimiento de los requerimientos de la vida adulta.

4. En relación con el juego como aprendizaje, resulta inherente al mismo la puesta a prueba de hipótesis e inferencias que se contrastan con la práctica lúdica, ya sea sobre relaciones sociales o conocimientos declarativos propiamente tales. Del mismo modo, el juego provee oportunidades de construcción de mundos imaginarios que favorecen el pensamiento abstracto y la generación de guiones narrativos que exigen la producción lingüística y cognitiva.

\section{Referencias bibliográficas}

Borghi, B. Q. (2010). Educar en el 0 3. Barcelona: Graó.

Borghi, B. Q. (2012). Una escuela infantil para el bienestar: 8 lineas de acción para una escuela 0 6 de calidad. Santiago de Chile: Universidad de Las Américas y Santo Tomás.

Fernández-Espejo, E. (2000). ¿Cómo funciona el nucleus accumbens? RevNeurol, $30,845-849$. 
Garvey, C. (1985). El juego infantil. Madrid: Morata.

Huizinga, J. (2012). Homo Ludens. Madrid: Alianza.

Michnick, R., Hirsh-Pasek, K. \& Singer, D. (2006). Play = Learning. How play motivates and enhances children's cognitive and social - emotional growth. New York: Oxford University Press.

Moreno, A. (2017). Colonialidad del conocimiento e infancia lúdica: rescatando los saberes infantiles desde el decir/hacer de los niños, en M. Mendoza y A. Moreno (eds.), Infancia, juego y corporeidad. Una mirada al aprendizaje desde el sur global (pp. 13-30). Santiago de Chile: Ediciones Junji.

Neuman, S. \& Roskos, K. (I992). Literacy objects as cultural tools: Effects on children's literacy behaviors during play. Reading Research Quarterly, 27, 203-223.

Piaget, J. (1990). La formación del símbolo en el niño. Buenos Aires: Fondo de Cultura Económica.

Platón. La República. Recuperado de http://www.bibliotecasvirtuales.com/ biblioteca/otrosautoresdelaliteraturauniversal/platon/larepublica/ index.asp

Smith, P. K. (2010). Children and play. Wiley- Blackwell.

Toro, S. y Sabogal, A. (2017). Motricidad, juego y aprendizaje encarnado, M. Mendoza y A. Moreno (eds.), Infancia, juego y corporeidad. Una mirada al aprendizaje desde el sur global (pp. 3 I-64). Santiago de Chile: Ediciones Junji.

Varela, F., Thompson, E. y Rosch, E. (I992). De Cuerpo Presente. Barcelona: Gedisa.

Vigotsky, L. S. (I966 [1933]). Play and its role in the mental development of the child. Voprosy Psikbologii, 12, 62-76. 